\title{
Influence of blood prostate specific antigen levels at age 60 on benefits and harms of prostate cancer screening: population based cohort study
}

\author{
(c) $(1)$ (8) OPEN ACCESS
}

\author{
Sigrid Carlsson research fellow ${ }^{12}$, Melissa Assel assistant research biostatistician ${ }^{3}$, Daniel Sjoberg \\ research biostatistician ${ }^{3}$, David Ulmert research fellow ${ }^{2}$, Jonas Hugosson attending urologist, \\ professor $^{1}$, Hans Lilja attending clinical chemist, professor ${ }^{2467}$, Andrew Vickers attending \\ biostatistician $^{3}$
}

${ }^{1}$ Department of Urology, Institute of Clinical Sciences, Sahlgrenska Academy at University of Gothenburg, Sweden; ${ }^{2}$ Department of Surgery (Urology service), Memorial Sloan-Kettering Cancer Center, New York, NY, USA; ${ }^{3}$ Department of Epidemiology and Biostatistics, Memorial Sloan-Kettering Cancer Center, New York, NY 10065, USA; ${ }^{4}$ Departments of Laboratory Medicine and Medicine (GU-Oncology), Memorial Sloan-Kettering Cancer Center, New York, NY, USA; ${ }^{5}$ Nuffield Department of Surgical Sciences, University of Oxford, Oxford, UK; ${ }^{6}$ Institute of Biomedical Technology, University of Tampere, Tampere, Finland; ${ }^{7}$ Department of Laboratory Medicine in Malmö, Lund University, Malmö, Sweden

\begin{abstract}
Objective To determine the relative risks of prostate cancer incidence, metastasis, and mortality associated with screening by serum prostate specific antigen (PSA) levels at age 60.

Design Population based cohort study.

Setting General male population of Sweden taking part in a screening trial in Gothenburg or participating in a cardiovascular study, the Malmö Preventive Project.

Participants The screened group consisted of 1756 men aged 57.5-62.5 participating in the screening arm of the Gothenburg randomized prostate cancer screening trial since 1995. The unscreened group consisted of 1162 men, born in 1921, participating in the Malmö Preventive Project, with PSA levels measured retrospectively in stored blood samples from 1981.

Intervention PSA screening versus no screening.

Main outcome measures Incidence rate ratios for the effect of screening on prostate cancer diagnosis, metastasis, and death by PSA levels at age 60.

Results The distribution of PSA levels was similar between the two cohorts. Differences in benefits by baseline PSA levels were large. Among men with baseline levels measured, 71.7\% (1646/2295) had a PSA level $<2 \mathrm{ng} / \mathrm{mL}$. For men aged 60 with PSA level $<2 \mathrm{ng} / \mathrm{mL}$, there was an increase in incidence of 767 cases per 10000 without a decrease in prostate cancer mortality. For men with PSA levels $\geq 2 \mathrm{ng} / \mathrm{mL}$, the reduction in cancer mortality was large, with only 23 men needing to be screened and six diagnosed to avoid one prostate cancer death by 15 years.
\end{abstract}

Conclusions The ratio of benefits to harms of PSA screening varies noticeably with blood PSA levels at age 60. For men with a PSA level $<1 \mathrm{ng} / \mathrm{mL}$ at age 60 , no further screening is recommended. Continuing to screen men with PSA levels $>2 \mathrm{ng} / \mathrm{mL}$ at age 60 is beneficial, with the number needed to screen and treat being extremely favourable. Screening men with a PSA level of $1-2 \mathrm{ng} / \mathrm{mL}$ is an individual decision to be based on a discussion between patient and doctor.

\section{Introduction}

Screening, by the use of simple tests in a healthy population, aims to identify those with who have a disease but no manifest symptoms. Patients with early stage prostate cancer are generally asymptomatic. When prostate cancer is palpable through digital rectal examination, the tumour has commonly grown through the prostate capsule. Screening and early detection by means of a blood test for prostate specific antigen (PSA) is a promising approach, since it provides the only way to identify asymptomatic men with prostate cancers that are curable. Such screening has been shown in large scale randomized trials to reduce mortality from the disease..$^{1-3}$ However, screening comes at a high cost in terms of screening participation and overdiagnosis: one estimate is that for every death from prostate cancer prevented by 11 years of follow-up, 1055 men need to be invited for screening and 37 cancers need to be detected. ${ }^{2} \mathrm{~A}$ possible strategy to improve the ratio of benefits to harms of PSA screening is to focus screening on men at highest risk of death from prostate cancer. 
One option is to use baseline PSA levels to stratify risk. There is a considerable literature showing that baseline PSA level is strongly predictive of subsequent outcomes from prostate cancer. ${ }^{4-9}$ For example, in a retrospective study based on the Malmö Preventive Project, 60 year old men who gave blood in 1981-2 as part of a cardiovascular study were followed to age 85. Rates of PSA testing were low. PSA levels assessed from stored blood samples were strongly predictive of subsequent prostate cancer mortality, with $90 \%$ of deaths occurring in men with PSA levels in the top $25 \%(>2 \mathrm{ng} / \mathrm{mL})$. Death from prostate cancer was rare $(0.2 \%)$ in men with PSA levels below the median value of about $1 \mathrm{ng} / \mathrm{mL}$. $^{5}$

These findings suggest that men with lower PSA levels at age 60 might be exempted from further screening. But recommendations for screening should be based on differences between screening and no screening groups, not risks within a group. For example, screening might still be justified if it reduced death rates to zero in the low PSA level group, a risk reduction of 2 per 1000 men screened.

We determined how baseline PSA level modifies the effects of PSA screening, for both benefits, in terms of reduced prostate cancer mortality, and harms, in terms of overdiagnosis. In this study screening is taken to include subsequent management, such as curative treatment for aggressive tumours and active surveillance for low risk disease. We hypothesised that the ratio between benefits and harms of screening in the screened Gothenburg group compared with the unscreened Malmö group would be superior in the group of men with the highest risk of prostate cancer mortality compared with the lower risk groups, by strata of baseline total PSA level.

\section{Methods}

\section{The Gothenburg screening study cohort}

The Gothenburg randomized prostate cancer screening trial is a population based study, approved by the ethical review committee at the University of Gothenburg in $1994 .{ }^{1}$ As of 31 December 1994, 32298 men were born between 1930 and 1944 (age 50-64) living in the city of Gothenburg, of whom 10000 were randomized to PSA screening and 10000 to control. Men randomized to screening were subsequently asked to consent to PSA testing. For the purposes of the current study, we included men screened at the first screening round 1995-96 (5855 attendees). We further restricted the cohort to 1756 screening attendees close to the age of 60 , defined as being between the ages of 57.5 and 62.5 years at baseline.

Outcomes ascertainment was as previously described. ${ }^{1}$ Briefly, we assessed cancer incidence through linkage with the screening database to the Swedish national cancer registry from 1 January 1995 to 31 December 2010. We assessed the endpoint of metastases from chart review and defined them as N1 (regional metastases), M1 (distant metastases), or a PSA concentration $>100 \mathrm{ng} / \mathrm{mL}$. For men without an event, the censoring date was the last visit to the urology or screening clinic when the patient with prostate cancer was known to be free of signs of metastasis (good health, no bone pain, PSA level not indicating metastases), or last follow-up for men free of disease.

A copy of the cause of death certificate was obtained for men who had died, and an independent blinded review committee determined the cause of death for men with a diagnosis of prostate cancer. Participants who emigrated or died of other causes were censored at the date of emigration or death. Participants who did not die were censored at their last documented follow-up date for vital status or the study end date, 31 December 2010.

\section{The Malmö Preventive Medicine Project cohort}

The Malmö cohort emerged from a unique subset from the Malmö Preventive Medicine Project cohort constituting of 1162 men born in 1921 in Malmö, Sweden, who provided blood samples at age 60 in 1981-82. As the PSA test was introduced in the mid-90s in Sweden, at which point the men in the cohort would have been aged 75 or older, this constitutes an unscreened population. Furthermore, the study cohort was highly representative, constituting $71 \%$ of the eligible population. Outcome ascertainment was predominately by chart review, with medical charts available for 101 of 126 men (80\%) with a diagnosis of prostate cancer and 72 of 87 men (83\%) who died after a diagnosis of prostate cancer. Cause of death was reviewed independently and blinded to baseline PSA level. ${ }^{7}$ This study was approved by the ethics committee at Lund University.

\section{Outcomes}

For both the Gothenburg and the Malmö cohorts, a metastasis event included metastases documented at the time of prostate cancer diagnosis as well as those documented subsequently. We defined men who died from prostate cancer without previous clinical documentation of metastasis as having a diagnosis of metastasis at the date of death. If patients had no previous documentation of cancer, they were defined as having a diagnosis of prostate cancer at the date of death in addition to metastasis.

\section{Laboratory measurements}

Laboratory methods were as previously detailed. ${ }^{17}$ In brief, men allocated to the screening arm of the Gothenburg study were invited for PSA testing every second year until they reached the upper age limit of 70 years. Blood was processed within three hours of venepuncture, frozen, and shipped frozen on dry ice for analyses within two weeks to Dr Lilja's laboratory at the Wallenberg Research Laboratories, Department of Laboratory Medicine, at Lund University in Malmö, Sweden. Men in the Malmö Preventive Project had total PSA assayed retrospectively in EDTA anticoagulated blood plasma that was stored at $-20^{\circ} \mathrm{C}$ for up to 27 years; during these conditions, total PSA

concentrations have been shown to be highly stable to long term storage and provide determinations corresponding to those had they been measured contemporaneously. ${ }^{10}$ Total PSA measurements blinded to outcome were performed at Dr Lilja's laboratory at the Wallenberg Research Laboratories at Lund University in Malmö using the dual label DELFIA Prostatus total/free PSA assay (Perkin-Elmer, Turku, Finland), ${ }^{11}$ calibrated in accordance to the change implemented in 2004 to reflect the World Health Organization 96/670 calibrator.

\section{Statistical analysis}

Our primary aim was to compare the number of cancers diagnosed, metastases prevented, and prostate cancer deaths prevented by baseline total PSA levels between screened and unscreened participants. Owing to the case-control design of the Malmö Preventive Project (three controls matched to each index case), blood was not sampled for some participants and we had to impute their PSA values, using methods previously described. ${ }^{7}$ We used the Kaplan-Meier method to calculate cumulative incidences for the outcomes of prostate cancer, prostate cancer metastases, overall mortality, and prostate cancer 
death by PSA levels at age 60 . The Nelson-Aalen method was used to plot the cumulative hazards for these events.

In some analyses no events occurred by 15 years in one group. To obtain confidence intervals for risk differences, we used binomial methods. Since there was greater censoring before 15 years in the Gothenburg arm, and because event rates were positively correlated with PSA levels, binomial methods bias against our hypothesis that the benefits of screening are small for men with lower PSA levels. As a sensitivity analysis, we calculated cumulative incidences adjusted for the competing risk of death from other causes using the Fine-Gray method. We calculated incidence rate ratios for the effect of screening on prostate cancer diagnosis, metastasis, and death.

To determine the benefits of continued screening after a baseline PSA measurement, we calculated the number of men needed to screen and the number of men needed to be diagnosed to prevent one man from developing metastatic disease or dying from prostate cancer by 15 years. We calculated the number needed to screen as the inverse of the absolute risk reduction between the screened and unscreened groups based on 15 years of follow-up. We calculated the number needed to be diagnosed as the inverse absolute risk reduction multiplied by the excess incidence of prostate cancer diagnosis in the screened group based on 15 years of follow-up. We used a conservative approach to calculate the number needed to be screened and number needed to be diagnosed, involving all men who were invited to screening in Gothenburg, whether or not they participated in all (or even any) follow-up screenings according to protocol.

We believe that the outcome of prostate cancer metastasis was followed more closely in the Gothenburg group, since these patients were attendees of a screening trial in regular contact with the urology clinic and access to tests such as bone scintigraphy. We therefore performed an additional sensitivity analysis where we defined Malmö participants without previous documentation of metastasis who died of prostate cancer between 15 and 17 years of follow-up as developing metastasis at 15 years of follow-up. All analyses were performed using Stata, version 12.0 (StataCorp, College Station, TX).

\section{Results}

Table $1 \Downarrow$ displays the characteristics of participants with a diagnosis of prostate cancer by group. Participants in the Malmö cohort were older and had more advanced disease at the time of prostate cancer diagnosis than participants in the Gothenburg cohort. Table $2 \Downarrow$ displays the cumulative incidence of each outcome at 15 years by baseline total PSA level and group. Risks of all endpoints increased with higher PSA levels. A total of 318 prostate cancers were diagnosed out of 1756 men in the Gothenburg group (cumulative incidence 19.6\%) compared with 63 out of 1162 men in the Malmö group (cumulative incidence $6.4 \%$ ). Of the 318 prostate cancers diagnosed in the Gothenburg group, 257 were detected through screening (81\%). Overall, there were 22 prostate cancer metastases (cumulative incidence $1.5 \%$ ) in the Gothenburg group compared with 20 (cumulative incidence 2.0\%) in the Malmö group, and 14 prostate cancer deaths (cumulative incidence $0.9 \%$ ) in the Gothenburg group compared with 16 (cumulative incidence $1.7 \%$ ) in the Malmö group.

Table $3 \Downarrow$ displays the 15 year outcome rates per 1000 person

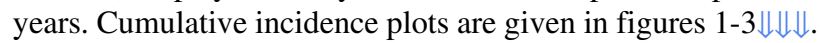
The distribution of PSA levels was similar between the two cohorts. The rate ratio of incident diagnosis, metastases, or death from prostate cancer was 3.67 (95\% confidence interval 2.79 to 4.89 ), 0.73 (0.38 to 1.42 ), and 0.57 (0.26 to 1.25 ), respectively, at 15 years among screened men compared with the unscreened men.

Table $4 \Downarrow$ shows the effects of screening on incidence, metastasis, and prostate cancer specific mortality separately for different PSA subgroups. Among men with baseline PSA levels measured, $71.7 \%(1646 / 2295)$ had a PSA level $<2 \mathrm{ng} / \mathrm{mL}$. The upper bound of the $95 \%$ confidence interval for risk reduction does not include any important benefit of screening among men aged 60 with PSA levels $<2 \mathrm{ng} / \mathrm{mL}$, especially given that the use of the binomial approximation in these groups biases findings towards screening. When men with PSA levels $<2$ $\mathrm{ng} / \mathrm{mL}$ are analysed as a single group, the increase in diagnosis incidence associated with screening was 767 (95\% confidence interval 565 to 970) and there was also an increase in the risk of death from prostate cancer of 49 (95\% confidence interval 5 to 74) per 10000 men.

Screening reduced prostate cancer mortality for men aged 60 with PSA levels $\geq 2 \mathrm{ng} / \mathrm{mL}$, with a number needed to screen of 23 and a number needed to diagnose of 6 to prevent one death from prostate cancer. A sensitivity analysis excluding cases diagnosed within one year of baseline venepuncture in both the Malmö and the Gothenburg cohorts did not change our conclusions (PSA level $\geq 2 \mathrm{ng} / \mathrm{mL}$ : number needed to screen of 16 and number needed to diagnose of 3 ).

Among those with baseline total PSA levels $\geq 3 \mathrm{ng} / \mathrm{mL}$, the number needed to screen to prevent one death from prostate cancer was 17 men and the number needed to diagnose was 5 men. Screening reduced prostate cancer mortality also in men with PSA levels of 2-2.99 ng/mL, with a number needed to screen of 45 and a number needed to diagnose of 12 to prevent one death from prostate cancer.

The sensitivity analysis for differential follow-up of metastasis did not importantly affect results (PSA level $\geq 3 \mathrm{ng} / \mathrm{mL}$ : number needed to screen of 17 and number needed to diagnose of 5 , PSA level 2-2.99 ng/mL: number needed to screen of 45 and number needed to diagnose of 12). Only two participants in the Malmö cohort died of prostate cancer between 15 and 17 years without documentation of metastasis before 15 years but had baseline PSA levels of $2.04 \mathrm{ng} / \mathrm{mL}$ and $3.28 \mathrm{ng} / \mathrm{mL}$. A sensitivity analysis using competing risk methods also failed to modify estimates of number needed to screen or number needed to diagnose (PSA level $\geq 3 \mathrm{ng} / \mathrm{mL}$ : number needed to screen of 19 and number needed to diagnose of 5, PSA level 2-2.99 ng/mL: number needed to screen of 57 and number needed to diagnose of 14).

\section{Discussion}

In the current study, we noted clear differences in the effects of PSA screening depending on the baseline PSA level at age 60 . Screening led to large reductions in prostate cancer mortality in the $26 \%$ (767/2918) of men with PSA levels $\geq 2 \mathrm{ng} / \mathrm{mL}$, with relatively few men needing to be screened and diagnosed to prevent one death from prostate cancer. We saw that men with a baseline PSA level $<1 \mathrm{ng} / \mathrm{mL}$ at age 60 constitute a low risk group for 15 year risk of metastasis and prostate cancer mortality. Men with a PSA level of 1-2 ng/mL might be considered a grey area. In all, men with a baseline PSA level $<2 \mathrm{ng} / \mathrm{mL}$ correspond to approximately three quarters of 60 year olds. Thus the balance between harms and benefits of PSA screening could be optimised if focus shifted to screening men at highest risk. Continuing to screen men aged 60 with PSA levels $<1$ and $1-2 \mathrm{ng} / \mathrm{mL}$ would possibly lead to overdiagnosis, with little, if any, effect of screening on preventing metastasis 
and death from prostate cancer. As such, exempting men aged 60 or more with PSA levels in these ranges from further rescreening would reduce their risk of overdiagnosis without compromising the risk of avoiding preventable metastasis of prostate cancer death for most men. Long term risk of cancer metastasis and death was low in men with PSA levels $<2 \mathrm{ng} / \mathrm{mL}$, in itself a reason to limit screening in this group.

Several findings support the use of the Malmö cohort as control. The point estimate of a relative risk of 0.57 for prostate cancer mortality at 15 years was consistent with that reported from the Gothenburg trial using the randomized no screening arm as control ( 0.56 at 14 years). ${ }^{1}$ The corresponding relative risks for a diagnosis of prostate cancer were 3.65 and $1.64,{ }^{1}$ respectively. The increased relative risk in this study possibly reflects both contamination in the Gothenburg control group and cancers detected for cause. Though the exact contamination in the Gothenburg control group during the course of the trial is difficult to assess, it is estimated that more than 1 in 3 men of screening age in Sweden has had a PSA test between 2000 and 2007. ${ }^{12} 13$

\section{Comparison with other studies}

Our findings are similar to others in the literature. Van Leeuwen and colleagues compared the benefits of screening by baseline PSA level in a screened group in the European Randomized trial of Screening for Prostate Cancer (ERSPC), of which the Gothenburg study is a component, with a non-screened population in Northern Ireland. For men with a PSA level between 0.0 and $1.99 \mathrm{ng} / \mathrm{mL}$ at study entry, the number needed to screen was extraordinarily high at 24 642, and the number needed to diagnose was similarly high, at 724 , making it hard to justify repeat screening for men with these levels..$^{14} \mathrm{~A}$ separate study on the Rotterdam branch of the ERSPC confirms the strong prognostic value of a baseline PSA level. Bul and colleagues reported that men aged 55-74 years who had a PSA level $<3 \mathrm{ng} / \mathrm{mL}$ at baseline were 10 times less likely to die from prostate cancer over the next 15 years than men with a higher PSA level $(\geq 3 \mathrm{ng} / \mathrm{mL})$. Those with a baseline PSA of $<1 \mathrm{ng} / \mathrm{mL}$ had a small risk of dying from the disease $(0.04 \%) .{ }^{15}$ Both estimates are comparable to those reported here.

It might be questioned why some men died of prostate cancer despite screening. Excluding six men whose diagnosis followed their first PSA screen, many of whom already had advanced disease, eight men in the screening group subsequently died. All but one had a PSA level $>1.0 \mathrm{ng} / \mathrm{mL}$ at baseline. Four men attended several screening visits following the screening protocol (complete attendees) and had PSA detected cancers, though one had a contraindication to prostate biopsy. One man followed the protocol but was diagnosed after termination of screening owing to reaching the upper age limit of 70 years. Another participated at irregular intervals, and two men each had one PSA measurement only. All but one man, who had intermediate risk disease, presented with high risk or locally advanced disease at diagnosis. This illustrates that risk stratification is important, as some men will probably need more intense and prolonged screening whereas many men have an extremely low risk and might stop screening at an early age.

\section{Strengths and limitations of this study}

A limitation of the present study is that 15 years may be too short a follow-up given the long clinical course of prostate cancer, especially given the moderate sample size (1756 men in Gothenburg $v 1162$ men in Malmö), which limited the number of events. Yet while this may explain the failure to find statistically significant overall differences in prostate cancer mortality, most of the confidence intervals for the PSA subgroups are sufficiently narrow to exclude alternative clinical implications. For example, for men with PSA concentrations between 1.00 and $1.99 \mathrm{ng} / \mathrm{mL}$, the lower bound of the $95 \%$ confidence interval for increase in cancer diagnoses per 10000 men screened is 1101, an important excess incidence.

With respect to the application of our findings to populations more racially diverse than that of Sweden, such as the US population, we accept that absolute risks of prostate cancer incidence and mortality may differ. For instance, aggressive prostate cancer is more common in African-Americans than in white men. ${ }^{16}$ However, our results focus on relative differences between PSA subgroups. Evidence that the prognostic value of PSA level is noticeably lower in non-white racial groups is lacking. Indeed, one study of a US population concluded that "there were no important or statistically significant differences in the performance [of PSA] by race." 17

Further replication is warranted, comparing screened and unscreened populations, for which data on PSA levels are available.

\section{Conclusions and implications}

Randomized trials have reported that the benefits of PSA, in terms of reduced mortality, come at a relatively high cost, considering the number of men who needed to be screened and diagnosed to prevent one death from prostate cancer. Here we report that these estimates are an average of two extremes for different PSA subgroups. The benefits of screening the high risk group of men with a PSA concentration $\geq 2 \mathrm{ng} / \mathrm{mL}$, constituting close to a quarter of the population, are large, whereas for men with a PSA concentration $<1 \mathrm{ng} / \mathrm{mL}$ at age 60 , PSA screening will lead to overdiagnosis without a mortality benefit. Therefore, for men with a PSA concentration $<1 \mathrm{ng} / \mathrm{mL}$ at age 60 , no further screening is recommended.

\section{The Gothenburg trial is registered as Current Controlled Trials} ISRCTN54449243.

Contributors: AV, $\mathrm{HL}, \mathrm{JH}$, and $\mathrm{SC}$ conceived and designed the study. $\mathrm{JH}, \mathrm{SC}, \mathrm{DU}$, and $\mathrm{HL}$ acquired the data. MA, AV, SC, JH, DS, and $\mathrm{HL}$ analysed the data. All authors interpreted the data. SC, MA, and AV drafted the manuscript. All authors critically revised the manuscript for important intellectual content and approved the submitted version. MA, AV, DS, and SC carried out the statistical analysis. $\mathrm{HL}, \mathrm{JH}$, and SC obtained funding. $\mathrm{AV}, \mathrm{HL}$, and $\mathrm{JH}$ were involved in administrative, technical, or material support. AV had full access to all the data in the study and takes responsibility for the integrity of the data and the accuracy of the data analysis. AV is guarantor.

Funding: SC was supported by grants from the Swedish Cancer Society, the Sweden America Foundation, the Swedish Council for Working Life and Social Research, the Swedish Society for Medical Research, and the Swedish Prostate Cancer Foundation. DU was supported by the David H Koch Prostate Cancer Foundation Young Investigator Award, The Tegger Foundation, and The Bertha Kamprad Foundation. This work was further supported by the National Cancer Institute (R01CA160816, R01 CA175491, and P50-CA92629), the Sidney Kimmel Center for Prostate and Urologic Cancers, and David H Koch through the Prostate Cancer Foundation, the National Institute for Health Research Oxford Biomedical Research Centre programme, Swedish Cancer Society (project No 11-0624), a FiDiPro-programme award from TEKES in Finland, and Fundacion Federico. Role of the funding source: none.

Competing interests: All authors have completed the ICMJE uniform disclosure form at www.icmje.org/coi_disclosure.pdf (available on 


\section{What is already known on this topic}

Screening for prostate cancer with prostate specific antigen (PSA) can reduce prostate cancer mortality

The ratio of benefits to harms of current screening practices is questionable, with a high number needed to be screened and diagnosed to prevent one death

Risk of prostate cancer mortality is low in men with PSA levels $<1-2 \mathrm{ng} / \mathrm{mL}$; whether prostate cancer mortality can be importantly reduced by continued screening in this group of men is not known

\section{What this study adds}

Prior estimates of screening have been an average of two very different subgroups based on the PSA level at age 60 We recommend that screening after the age of 60 should focus on the high risk group of men with PSA levels $\geq 2 \mathrm{ng} / \mathrm{mL}$; among which only 23 men need to be screened to prevent one prostate cancer death at 15 years, and only six diagnosed

request from the corresponding author) and declare: no support from any organisation for the submitted work; no financial relationships with any organisations that might have an interest in the submitted work in the previous three years; $\mathrm{HL}$ holds patents for free prostate specific antigen (PSA), kallikrein related peptidase 2, and intact PSA assays, and along with $\mathrm{AV}$, is named as co-inventor on a patent application for intact/nicked PSA assays and for a statistical method for predicting the result of a prostate biopsy.

Ethical approval: The Gothenburg randomized prostate cancer screening trial was approved by the ethical review committee at Gothenburg University. The Malmö Preventive Medicine Project cohort was approved by the ethics committee at Lund University, Sweden with official record No 85/2004 for epidemiological studies.

Data sharing: A full dataset of the Malmö component of this study is available from the authors on request, and from the Swedish national data archive (www.ssd.gu.se/en). These data can be used only for replication of the analyses published in this paper or for private study. Express written permission must be sought from the authors for any other data use. Data from the Gothenburg randomized trial are not available to outside investigators.

Transparency: The senior author (AV) affirms that the manuscript is an honest, accurate, and transparent account of the study being reported; that no important aspects of the study have been omitted; and that any discrepancies from the study as planned (and, if relevant, registered) have been explained.

1 Hugosson J, Carlsson S, Aus G, Bergdahl S, Khatami A, Lodding P, et al. Mortality results from the Göteborg randomised population-based prostate-cancer screening trial. Lancet Oncol 2010;11:725-32.

2 Schroder FH, Hugosson J, Roobol MJ, Tammela TL, Ciatto S, Nelen V, et al. Prostate-cancer mortality at 11 years of follow-up. N Engl J Med 2012;366:981-90.

3 Schröder FH, Hugosson J, Roobol MJ, Tammela TL, Ciatto S, Nelen V et al. Screening and prostate-cancer mortality in a randomized European study. N Engl J Med 2009;360:1320-8.

4 Loeb S, Carter HB, Catalona WJ, Moul JW, Schroder FH. Baseline prostate-specific antigen testing at a young age. Eur Urol 2012;61:1-7.
5 Lilia H, Ulmert D, Bjork T, Becker C, Serio AM, Nilsson JA, et al. Long-term prediction of prostate cancer up to 25 years before diagnosis of prostate cancer using prostate kallikreins measured at age 44 to 50 years. J Clin Oncol 2007;25:431-6.

6 Lilja H, Cronin AM, Dahlin A, Manjer J, Nilsson PM, Eastham JA, et al. Prediction of significant prostate cancer diagnosed 20 to 30 years later with a single measure of prostate-specific antigen at or before age 50. Cancer 2011:117:1210-9.

7 Vickers AJ, Cronin AM, Bjork T, Manjer J, Nilsson PM, Dahlin A, et al. Prostate specific antigen concentration at age 60 and death or metastasis from prostate cancer: case-control study. BMJ 2010;341:c4521.

8 Whittemore AS, Cirillo PM, Feldman D, Cohn BA. Prostate specific antigen levels in young adulthood predict prostate cancer risk: results from a cohort of black and white Americans. J Urol 2005;174:872-6.

9 Zhu X, Albertsen PC, Andriole GL, Roobol MJ, Schroder FH, Vickers AJ. Risk-based prostate cancer screening. Eur Urol 2012;61:652-61.

10 Ulmert D, Becker C, Nilsson JA, Piironen T, Björk T, Hugosson J, et al. Reproducibility and accuracy of measurements of free and total prostate-specific antigen in serum vs plasma after long-term storage at -20 degrees C. Clin Chem 2006;52:235-9.

11 Mitrunen K, Pettersson K, Piironen T, Björk T, Lilja H, Lövgren T. Dual-label one-step immunoassay for simultaneous measurement of free and total prostate-specific antigen concentrations and ratios in serum. Clin Chem 1995;41:1115-20.

12 Bratt O, Berglund A, Adolfsson J, Johansson JE, Tornblom M, Stattin P. Prostate cancer diagnosed after prostate-specific antigen testing of men without clinical signs of the disease: a population-based study from the National Prostate Cancer Register of Sweden. Scand J Urol Nephrol 2010;44:384-90.

13 Jonsson H, Holmstrom B, Duffy SW, Stattin P. Uptake of prostate-specific antigen testing for early prostate cancer detection in Sweden. Int J Cancer 2011;129:1881-8.

14 Van Leeuwen PJ, Connolly D, Tammela TL, Auvinen A, Kranse R, Roobol MJ, et al. Balancing the harms and benefits of early detection of prostate cancer. Cancer 2010;116:4857-65.

15 Bul M, van Leeuwen PJ, Zhu X, Schröder FH, Roobol MJ. Prostate cancer incidence and disease-specific survival of men with initial prostate-specific antigen less than $3.0 \mathrm{ng} / \mathrm{ml}$ who are participating in ERSPC Rotterdam. Eur Urol 2011;59:498-505.

16 Schwartz KL, Crossley-May H, Vigneau FD, Brown K, Banerjee M. Race, socioeconomic status and stage at diagnosis for five common malignancies. Cancer Causes Control 2003;14:761-6.

17 Whittemore AS, Lele C, Friedman GD, Stamey T, Vogelman JH, Orentreich N Prostate-specific antigen as predictor of prostate cancer in black men and white men. $J$ Natl Cancer Inst 1995;87:354-60.

\section{Accepted: 17 March 2014}

\section{Cite this as: BMJ 2014;348:g2296}

This is an Open Access article distributed in accordance with the Creative Commons Attribution Non Commercial (CC BY-NC 3.0) license, which permits others to distribute, remix, adapt, build upon this work non-commercially, and license their derivative works on different terms, provided the original work is properly cited and the use is non-commercial. See: http://creativecommons.org/licenses/by-nc/3.0/. 


\section{Tables}

Table 1 | Characteristics of participants with a diagnosis of prostate cancer within 15 years of follow-up by cohort. Values are numbers (percentages) unless stated otherwise

\begin{tabular}{|c|c|c|c|}
\hline Characteristics & Malmö cohort $(n=63)$ & Gothenburg cohort $(n=318)$ & $P$ value ${ }^{*}$ \\
\hline Median (interquartile range) total PSA level at baseline $(\mathrm{ng} / \mathrm{mL})$ & $3.89(2.04-6.88)$ & $2.67(1.73-4.45)$ & 0.024 \\
\hline Unknown & $1(0.3)$ & $0(0.0)$ & \\
\hline \multicolumn{4}{|l|}{ T stage: } \\
\hline $\mathrm{T} 1$ & $3(4.8)$ & $0(0)$ & $<0.001$ \\
\hline $\mathrm{T} 1 \mathrm{~A}$ & $4(6.3)$ & $4(1.3)$ & \\
\hline $\mathrm{T} 1 \mathrm{~B}$ & $2(3.2)$ & $1(0.3)$ & \\
\hline $\mathrm{T} 1 \mathrm{C}$ & $3(4.8)$ & $230(72)$ & \\
\hline $\mathrm{T} 2$ & $26(41)$ & $66(21)$ & \\
\hline $\mathrm{T} 3$ & $13(21)$ & $9(2.8)$ & \\
\hline $\mathrm{T} 4$ & $3(4.8)$ & $3(0.9)$ & \\
\hline $\mathrm{TX}$ & $9(14)$ & $4(1.3)$ & \\
\hline Unknown & $0(0)$ & $1(0.3)$ & \\
\hline \multicolumn{4}{|l|}{ Lymph node metastases at diagnosis: } \\
\hline No & $8(13)$ & $0(0)$ & 0.004 \\
\hline $\mathrm{N} 1$ & $0(0)$ & $2(0.6)$ & \\
\hline NX & $55(87)$ & $1(0.3)$ & \\
\hline Unknown & $0(0)$ & $315(99)$ & \\
\hline Distant metastases at diagnosis & $6(9.5)$ & $6(1.9)$ & 0.007 \\
\hline \multicolumn{4}{|l|}{ Biopsy score: } \\
\hline GS 2-6 & - & $246(77)$ & \\
\hline GS 7 & - & $62(19)$ & \\
\hline GS 8-10 & - & $10(3.1)$ & \\
\hline \multicolumn{4}{|l|}{ WHO classification } \\
\hline WHO I & $16(25)$ & - & \\
\hline WHO II & $18(29)$ & - & \\
\hline WHO III & $7(11)$ & - & \\
\hline Median (interquartile range) age at diagnosis (years) & $70(66-73)$ & $66(62-68)$ & $<0.001$ \\
\hline Median (interquartile range) total PSA level near diagnosis $(\mathrm{ng} / \mathrm{mL})$ & $20.8(13.9-34.3)$ & $4.5(3.5-7.2)$ & $<0.001$ \\
\hline Unknown & $36(11.3)$ & $2(3.2)$ & \\
\hline
\end{tabular}

WHO=World Health Organization .

${ }^{*}$ Calculated using Wilcoxon rank sum test for continuous variables and Fisher's exact test for categorical variables. 
Table 2| Cumulative incidences (\%) during 15 year follow-up period by group and baseline prostate specific antigen (PSA) level

\begin{tabular}{|c|c|c|c|c|}
\hline \multirow[b]{3}{*}{ Outcome } & \multicolumn{4}{|c|}{ Cumulative incidence (\%) } \\
\hline & \multicolumn{4}{|c|}{ Baseline total PSA level $(\mathrm{ng} / \mathrm{mL})$} \\
\hline & $0-0.99$ & $1-1.99$ & 2-2.99 & $\geq 3$ \\
\hline \multicolumn{5}{|c|}{ Population based centile*: } \\
\hline Gothenburg cohort & $<39$ & $39-72$ & $72-83$ & $\geq 83$ \\
\hline Malmö cohort & $<44$ & $44-76$ & $76-86$ & $\geq 86$ \\
\hline \multicolumn{5}{|c|}{ Prostate cancer diagnosis: } \\
\hline Gothenburg cohort & 3.6 & 16.6 & 37.5 & 49.5 \\
\hline Malmö cohort & 1.9 & 2.0 & 11.7 & 25.2 \\
\hline \multicolumn{5}{|c|}{ Prostate cancer metastasis: } \\
\hline Gothenburg cohort & 0.4 & 1.0 & 0.6 & 5.6 \\
\hline Malmö cohort & 0.0 & 0.3 & 2.2 & 11.4 \\
\hline \multicolumn{5}{|l|}{ Prostate cancer death: } \\
\hline Gothenburg cohort & 0.2 & 0.8 & 0.0 & 3.4 \\
\hline Malmö cohort & 0.0 & 0.0 & 2.2 & 9.5 \\
\hline
\end{tabular}

Cumulative incidences were calculated using the Kaplan-Meier method.

${ }^{*}$ Centiles can be used to find the percentage of men in each PSA category at age 60 . 
Table 3| Incidence rates, rate differences, and rate ratios for 15 year follow-up per 1000 person years

\begin{tabular}{|c|c|c|c|c|}
\hline \multirow[b]{2}{*}{ Prostate cancer outcomes } & \multicolumn{2}{|c|}{ Incidence rate $(95 \% \mathrm{Cl})$} & \multirow[b]{2}{*}{ IRD* $^{*}(95 \% \mathrm{CI})$} & \multirow[b]{2}{*}{ IRRT (95\% CI) } \\
\hline & Malmö cohort & Gothenburg cohort & & \\
\hline Diagnosis & 4.1 (3.2 to 5.3 ) & $15.2(13.6$ to 17.0$)$ & $11.1(9.1$ to 13.0$)$ & $3.67(2.79$ to 4.89$)$ \\
\hline Metastasis & $1.30(0.84$ to 2.01$)$ & 0.95 (0.63 to 1.45$)$ & $-0.35(-1.04$ to 0.35$)$ & 0.73 (0.38 to 1.42$)$ \\
\hline Mortality & 1.04 (0.63 to 1.69$)$ & $0.59(0.35$ to 1.00$)$ & $-0.44(-1.04$ to 0.15$)$ & $0.57(0.26$ to 1.25$)$ \\
\hline
\end{tabular}

*Incidence rate difference (IRD) was defined as incidence in Malmö cohort subtracted from incidence in Gothenburg cohort. †Incidence rate ratio (IRR) was defined as rate in Gothenburg cohort divided by rate in Malmö cohort. 
Table 4| Difference in risk between Malmö and Gothenburg cohorts per $\mathbf{1 0} 000$ men by baseline total prostate specific antigen (PSA) level based on 15 year follow-up

\begin{tabular}{|c|c|c|c|c|c|c|}
\hline \multirow[b]{3}{*}{ Prostate cancer outcomes } & \multicolumn{6}{|c|}{ Risk difference/10 000 men $(95 \% \mathrm{Cl})$} \\
\hline & \multicolumn{6}{|c|}{ Baseline total PSA level (ng/mL) } \\
\hline & $0-0.99$ & $1-1.99$ & $0-1.99$ & $2-2.99$ & $\geq 2$ & $\geq 3$ \\
\hline Increase in diagnosis & $171(-32$ to 374$)$ & 1462 (1101 to 1822$)$ & 767 (565 to 970$)$ & 2578 (1602 to 3554$)$ & 2485 (1797 to 3173$)$ & 2432 (1502 to 3362$)$ \\
\hline Decrease in metastasis & $-37(-70 \text { to } 11)^{*}$ & $-70(-182$ to 42$)$ & $-54(-112$ to 3$)$ & $158(-167$ to 483$)$ & 415 (30 to 799$)$ & $579(-15$ to 1172$)$ \\
\hline Decrease in death & $-17(-43 \text { to } 14)^{\star}$ & $-85(-138 \text { to }-2)^{\star}$ & $-49(-74 \text { to }-5)^{*}$ & $225(-68 \text { to } 425)^{*}$ & 453 (108 to 797$)$ & 604 (76 to 1131$)$ \\
\hline
\end{tabular}

Estimates rounded to nearest whole number.

${ }^{*}$ Confidence interval estimated using binomial methods. 


\section{Figures}

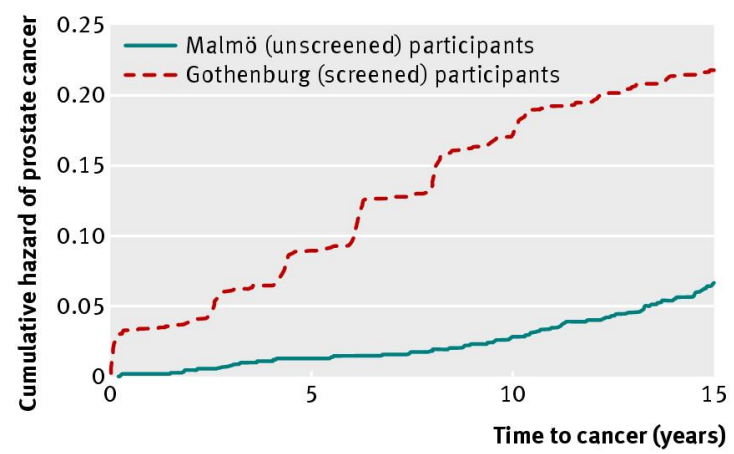

Fig 1 Cumulative risk of prostate cancer diagnosis

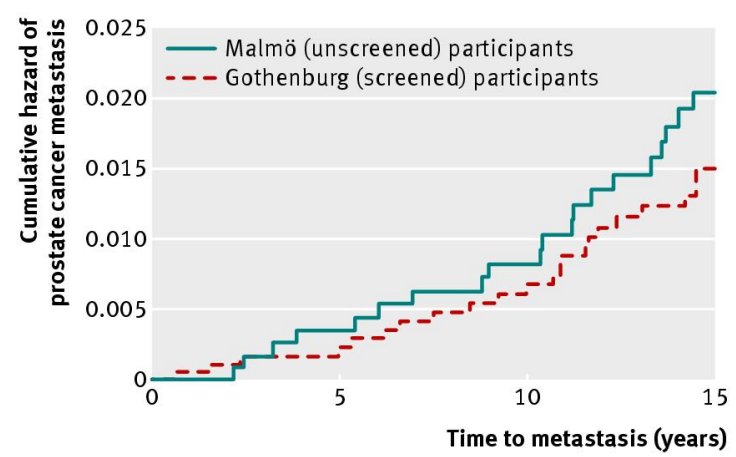

Fig 2 Cumulative risk of prostate cancer metastasis

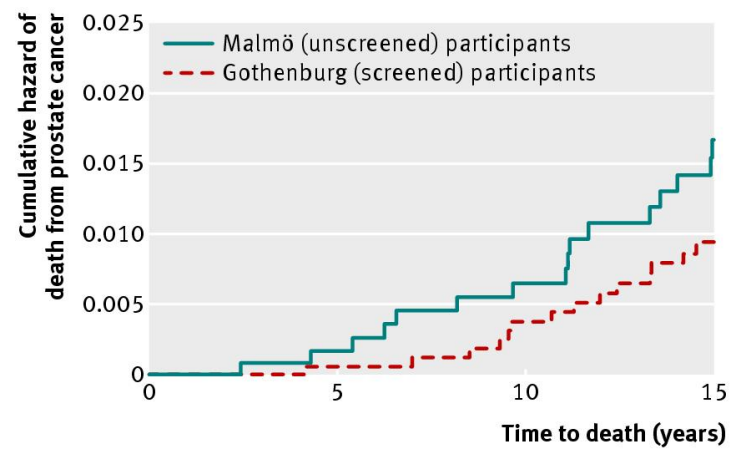

Fig 3 Cumulative risk of prostate cancer death 